\title{
An Ambiguous Case of Abdominal Pain Involving Renal Artery Thrombosis with Renal Infarction
}

Muhammad Shohel Almamun 1; Abrar Munir 1; Ali Riaz Raza 1;Sakina Koubeh 1; Hassan AbuZour 1; Hesham Kaawan1; Sarah Moore1; Ramneet Khurana1

1. Department of Medicine, North Manchester General Hospital ( Pennine Acute Hospitals), Delaunays Rd, Crumpsall, Manchester M8 5RB, UK.

Correspondence to Dr. Muhammad Shohel Almamun.

Email:malmamun@doctors.org.uk

Contact Tel: +44-161-720-2216/ +44-161-720-2316

Key words: Lactate dehydrogenase(LDH), Computed Tomography (CT), Glomerular filtration rate (GFR), C reactive protein(CRP), Angiotensin converting enzyme inhibitor (ACEI) Angiotensin receptor blocker (ARB), Kidney Urinary bladder( KUB), Dimercapto Succinic Acid (DMSA)

\section{Abstract}

Renal artery thrombosis is a sporadic serious clinical condition which potentially cause renal infarction. Diagnosis of renal infarction can be delayed or missed due to non specific clinical presentation and overlapping appearance of medical and surgical phenomena. Early diagnosis supported by biochemical and radiological findings while appropriate management potentially improve morbidity and mortality. Persistent abdominal or flank pain with raised LDH and proteinuria on background of thromboembolism risk factors supports the diagnosis. Despite the rarity of the disease rapid identification with prompt medical or endovascular intervention could prevent irreversible renal parenchymal damage.

\section{Introduction}

Renal artery thrombosis is rare incidence of $0.0007 \%$ but serious clinical condition which some times underestimated and misdiagnosed secondary to overlying similar medical and surgical cases (1). Renal infarction representing common disease condition results irreversible renal parenchymal damage reports in different case reports due to delayed or misdiagnosed. Renal artery thromboembolism causing persistent and unexplained flank pain or abdominal pain mimicking renal calculus and pyelonephritis. Therefore, raised LDH, haematuria, leucocytosis along with radiological modality of computed tomography supports diagnosis of early renal arterial thrombosis and infarction (2). In the context of inexplicable abdominal and flank pain emergency and acute physician should consider renal artery thrombosis in 
background of risk factors associated to this clinical scenario. In a situation of unusual and persistent abdominal or flank pain diagnostic evaluation for this clinical suspicion based on modalities of CT scan, scintigraphy, renal angiography and ultrasound (3). Embolism related to cardiac diseases and arrhythmia is a major cause but clinical manifestation substantially depends on unilateral or bilateral renal arterial involvement. In our case report renal artery thrombosis and renal infarction is associated with chronic atrial fibrillation and congestive cardiac failure.

\section{Case Report}

A 76 years old lady was admitted with confusion, low grade fever, lower abdominal, left flank and left iliac fossa pain for three days associated with nausea and vomiting. Her vomitus was brown in colour without any history of haematemesis or melena. She also complained of diarrhoea and low grade fever for two days. Diarrhoea was liquid to watery in consistency with variable colour mixed with mucus. She did not have any chest pain, short of breath, urinary frequency or dysuria. Her past medical history including mitral stenosis, breast cancer waiting for curative surgery, congestive cardiac failure, atrial fibrillation, permanent pacemaker ( PPM) and lumbar spine stenosis.

on examination her blood pressure was $130 / 65$, heart rate $98 / \mathrm{min}$, respiratory rate 18,Temperature 37.8 degree centigrade, saturation $98 \%$ on air. Chest sound noted left basal crepitation on auscultation, Heart sound was normal and abdomen tender on palpation in left flank and left iliac fossa but observed no guarding or rigidity. Initial blood results showed white cell count $1610^{*} 9 / \mathrm{L}$, Neutrophil $14.310^{*} 9 / \mathrm{L}$, CRP $4.4 \mathrm{mg} / \mathrm{L}$, Lactate 2.00 $\mathrm{mmol} / \mathrm{L}$, Lactate dehydrogenase $744 \mathrm{u} / \mathrm{L}$, INR 1.0,Urea $5.4 \mathrm{mmol} / \mathrm{L}$, Creatinine $124 \mathrm{umol} / \mathrm{L}$, GFR $35 \mathrm{~mL} / \mathrm{min}$, Amylase $71 \mathrm{u} / \mathrm{L}$, Bilirubin $21 \mathrm{umol} / \mathrm{L}$, ALP $96 \mathrm{u} / \mathrm{L}$, ALT $17 \mathrm{u} / \mathrm{L}$, Albumin $37 \mathrm{~g} / \mathrm{L}$. Urine protein creatine ratio 212.8 $\mathrm{mg} / \mathrm{mmol}$. On blood gas results $\mathrm{pH}$ was 7.49 , Base excess $3.6 \mathrm{mmol} / \mathrm{L}$ and bicarbonate $26.7 \mathrm{mmol} / \mathrm{L}$. and ECG known Atrial Fibrillation. Repeat blood results after 48 hours later revealed white cell count $31.610^{*} 9 / \mathrm{L}$, Neutrophil $27.410 * 9 / \mathrm{L}$, CRP $260 \mathrm{mg} / \mathrm{L}$, Urea $9.0 \mathrm{mmol} / \mathrm{L}$, Creatinine $150 \mathrm{umol} / \mathrm{L}$, GFR $28 \mathrm{~mL} / \mathrm{min}$, Bilirubin 25 umol/L, ALT $48 \mathrm{u} / \mathrm{L}$, ALP $76 \mathrm{u} / \mathrm{L}$. Albumin $25 \mathrm{~g} / \mathrm{L}$. CXR exhibited some inflammatory changes in left basal area but no evidence of consolidation and abdominal $X$-ray was unremarkable. Urine dipsticks showed protein and bloods. Urine microscopy and culture was negative. Initial differential diagnosis was Gastroenteritis, Diverticulitis. lower respiratory tract infection. Patient was treated with antibiotics, intravenous fluids, analgesia while with holding nephrotoxic medications but sign and symptoms of abdominal pain and left flank pain was persistent. Her presentation was not particularly clear regardless of documented feature of inflammatory response. Repeat bloods revealed deteriorating renal function and inflammatory markers despite appropriate antibiotic treatment. Patient was waiting for curative breast surgery for underlying breast cancer therefore on hold her anticoagulation warfarin. At this point patient's confirm diagnosis was in dilemma because no definitive diagnosis or source of infection was identified. Patient's symptoms of abdominal pain and tenderness was not improving despite antibiotics and analgesia while kidney function got worse 
progressively. At this moment surgical team was warranted to rule out underlying bowel obstruction or perforation Therefore, CT abdomen and pelvis was requested which incidentally confirmed minimal cortical enhancement of left kidney and left renal artery thrombosis. DMSA scan revealed minimal functional (17\%) left kidney compare $83 \%$ functional right kidney. KUB Vascular Ultrasound simultaneously established left renal artery thrombus. Recent echocardiography noted normal Ventricular function but Mitral valve was thickened / tethered leaflets with doming of both on opening with dilated both atrium. Radiological evidence revealed acute arterial occlusion of the left main renal artery with minimal perfusion of the left kidney. In this case theoretically it demonstrated like common scenario of thrombosis but practically many alternative clinical impressions was delivered in first instance. It was the CT imaging which incidentally identified the diagnosis followed by supportive biochemical evidence.

Consequently, patient case had been discussed with vascular surgeon for potential thrombolysis or embolectomy to prevent parenchymal damage. Anticoagulation with low molecular weight heparin was considered because atrial fibrillation with background of heart failure was the cause of thromboembolism. Renal specialist team delivered valuable input regarding management and follow up. Patient initial presentation had feature of sepsis with clinical diagnosis similar to diverticulitis, gastroenteritis, ischaemic bowel or even chest infection with element of renal impairment which was a very common scenarios in acute hospital settings. However, in chronic atrial fibrillation with warfarin or anticoagulation along with variable INR was routinely found in a medical ward. Nevertheless, this was unexpected for arterial thrombosis rather than venous thrombosis without any clinical suspicion. Presence of sepsis without any clinical feature of thrombosis it was really challenging to differentiate renal artery thrombosis and infarction which was life threatening. we were more focused on challenging clinical diagnosis rather than rarity of condition. In this case renal dialysis was not required because of early diagnosis of renal infarction and significant improvement of renal functional improvement. Screening test for vasculitis and hypercoagulable test was inconclusive. The confirm diagnosis was left renal artery thrombosis secondary to atrial fibrillation. 


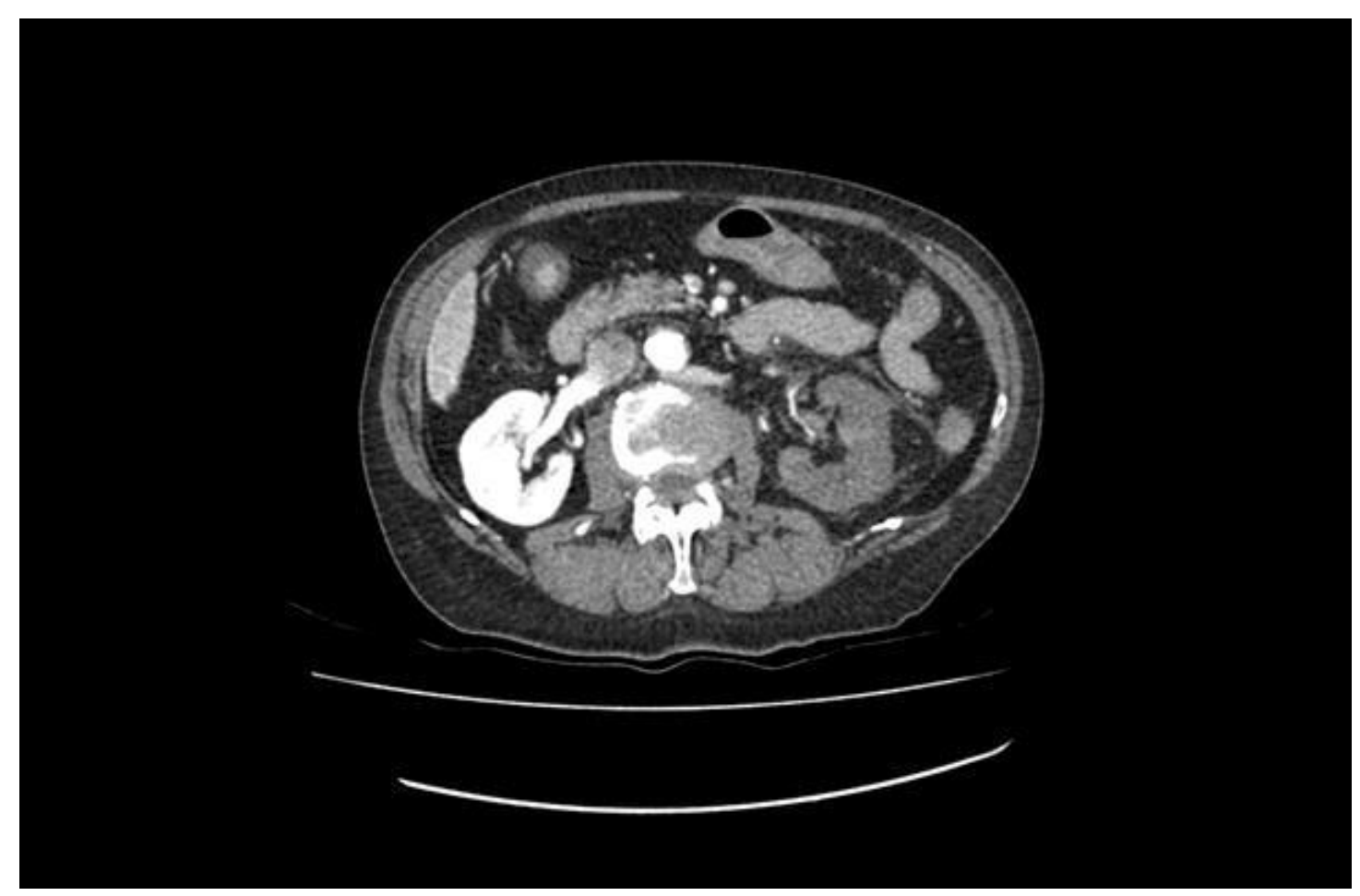

Figure 1: Coronal Contrast enhanced CT shows filling defect in left renal artery with patchy cortical enhancement in left kidney.

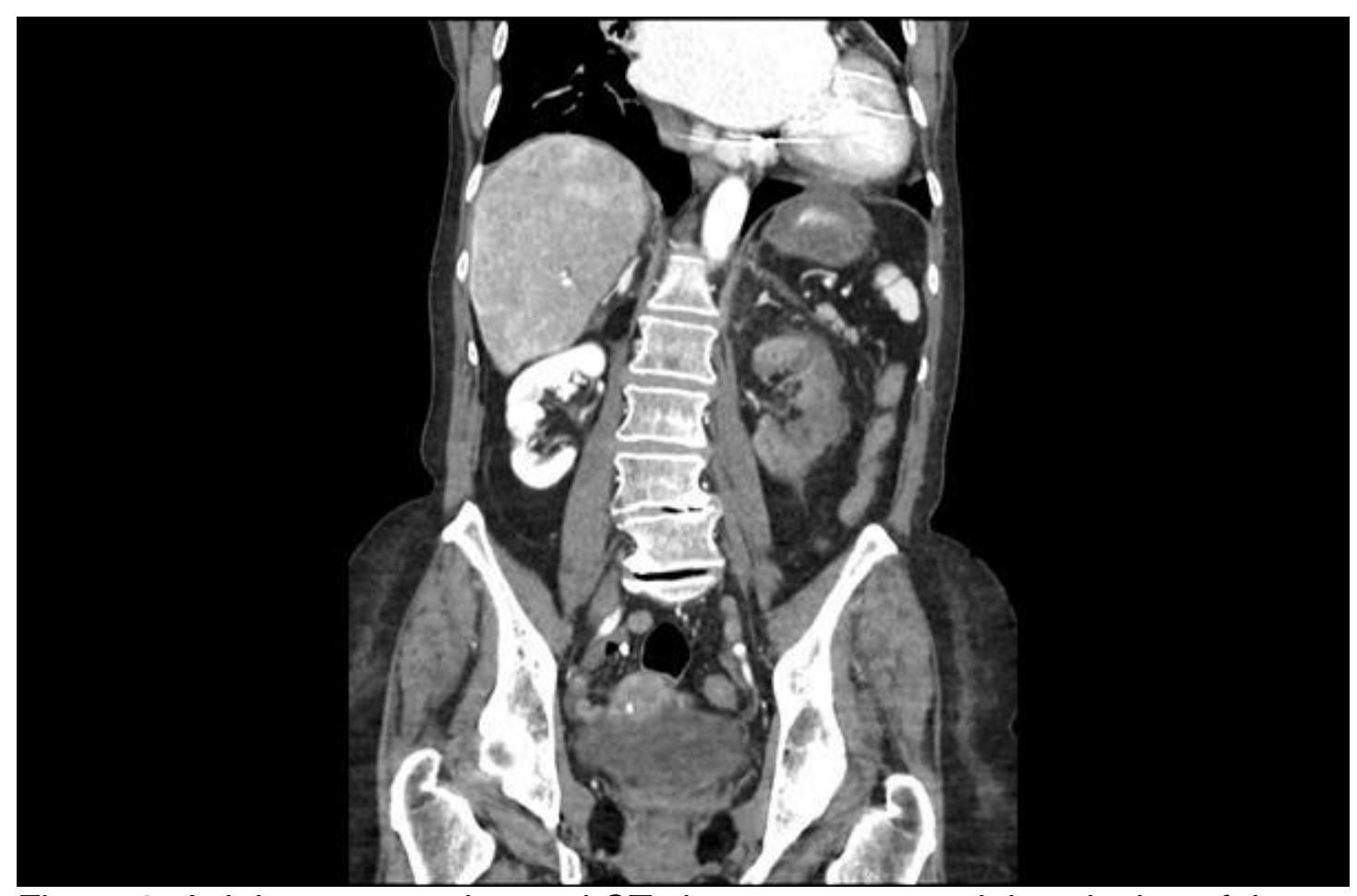

Figure 2: Axial contrast enhanced CT shows acute arterial occlusion of the left main renal artery with minimal perfusion of the left kidney. 


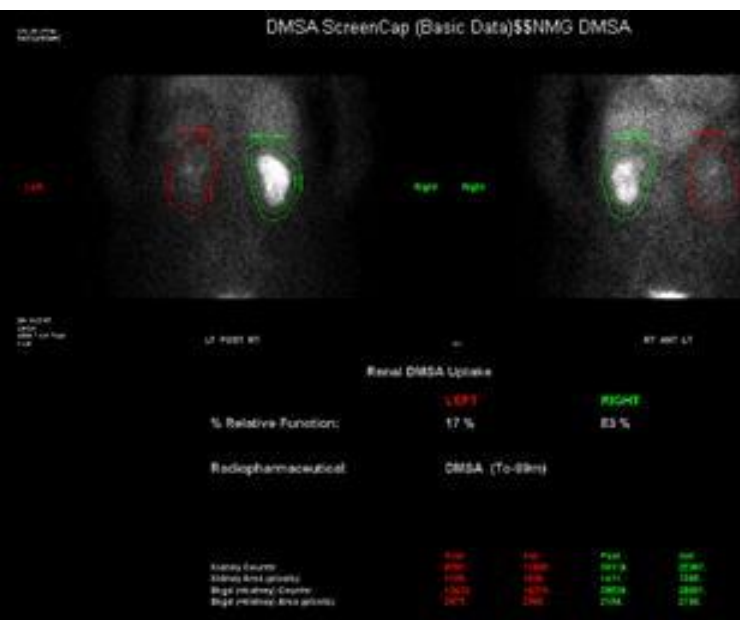

Figure: 3, DMSA Scan Poorly functioning left kidney (17\%)

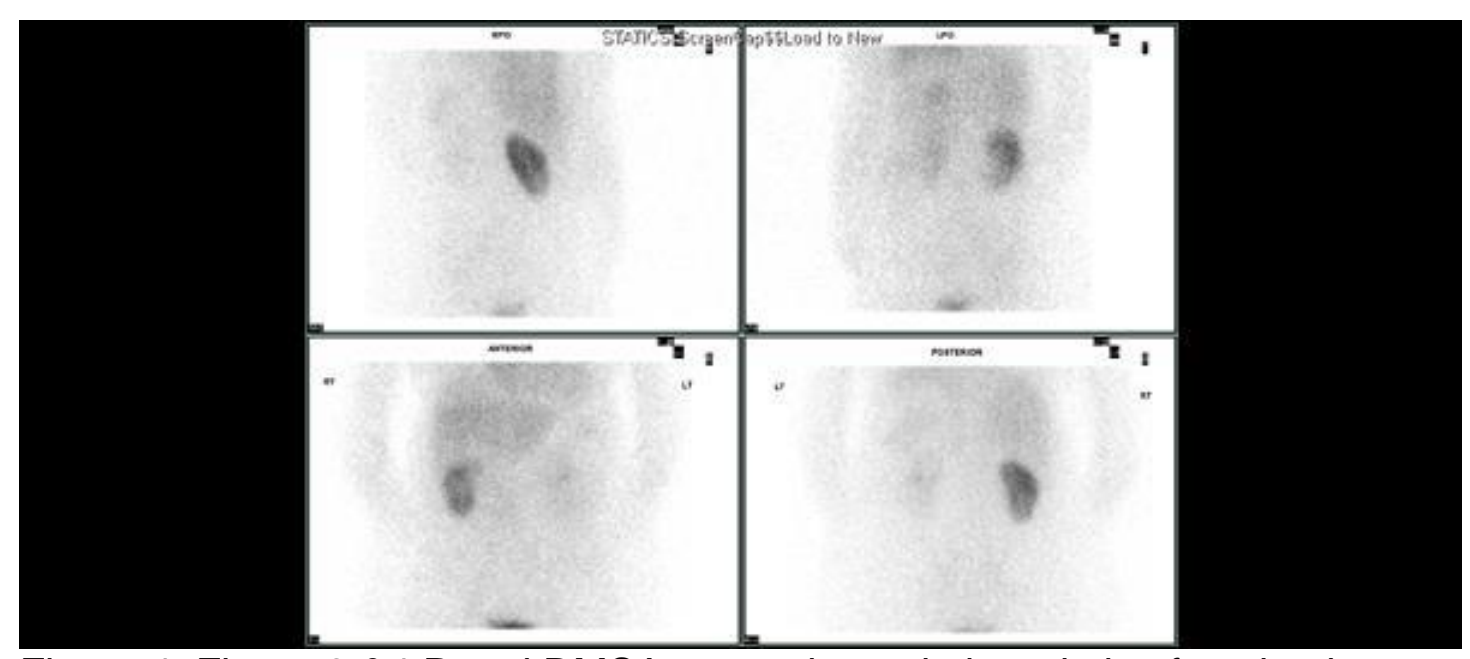

Figure 4: Figure 3 \&4 Renal DMSA scan showed the relative function is calculated to be left kidney $17 \%$ and right kidney $83 \%$. Indicative of Poorly functioning left kidney 


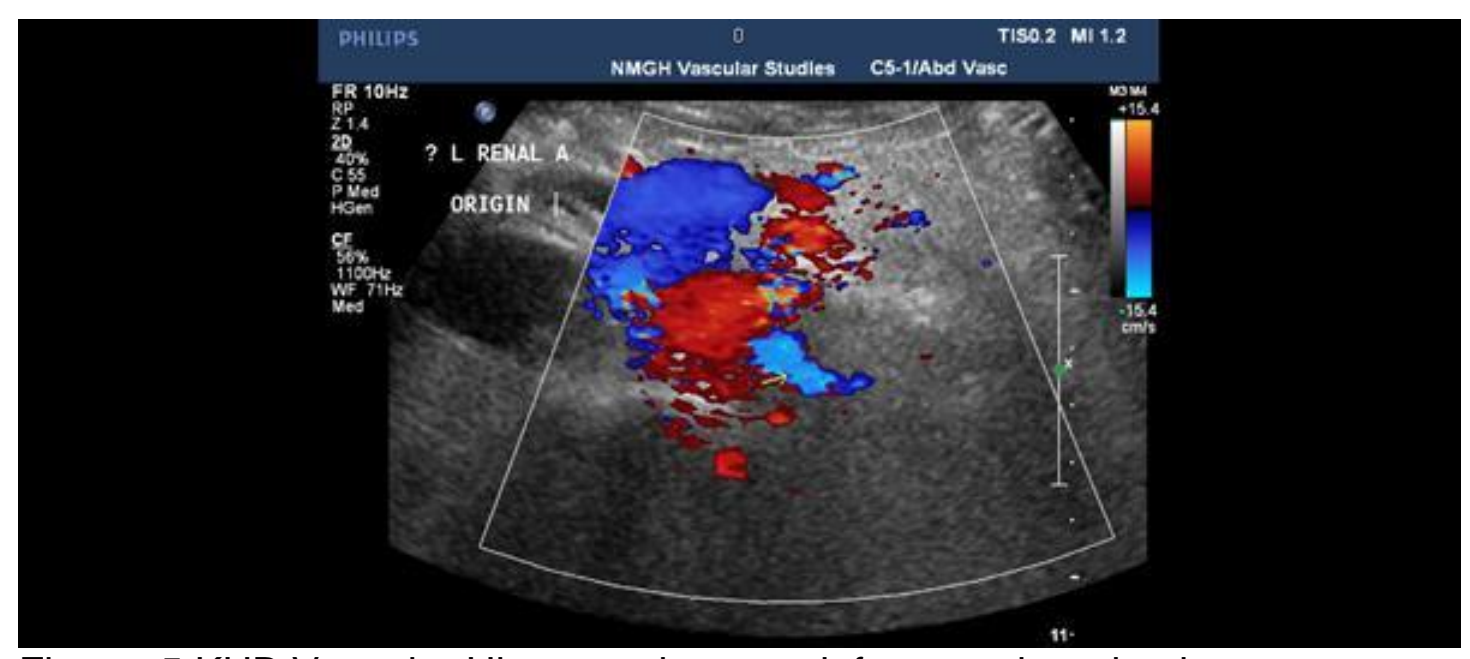

Figure: 5 KUB Vascular Ultrasound reports left artery thrombosis

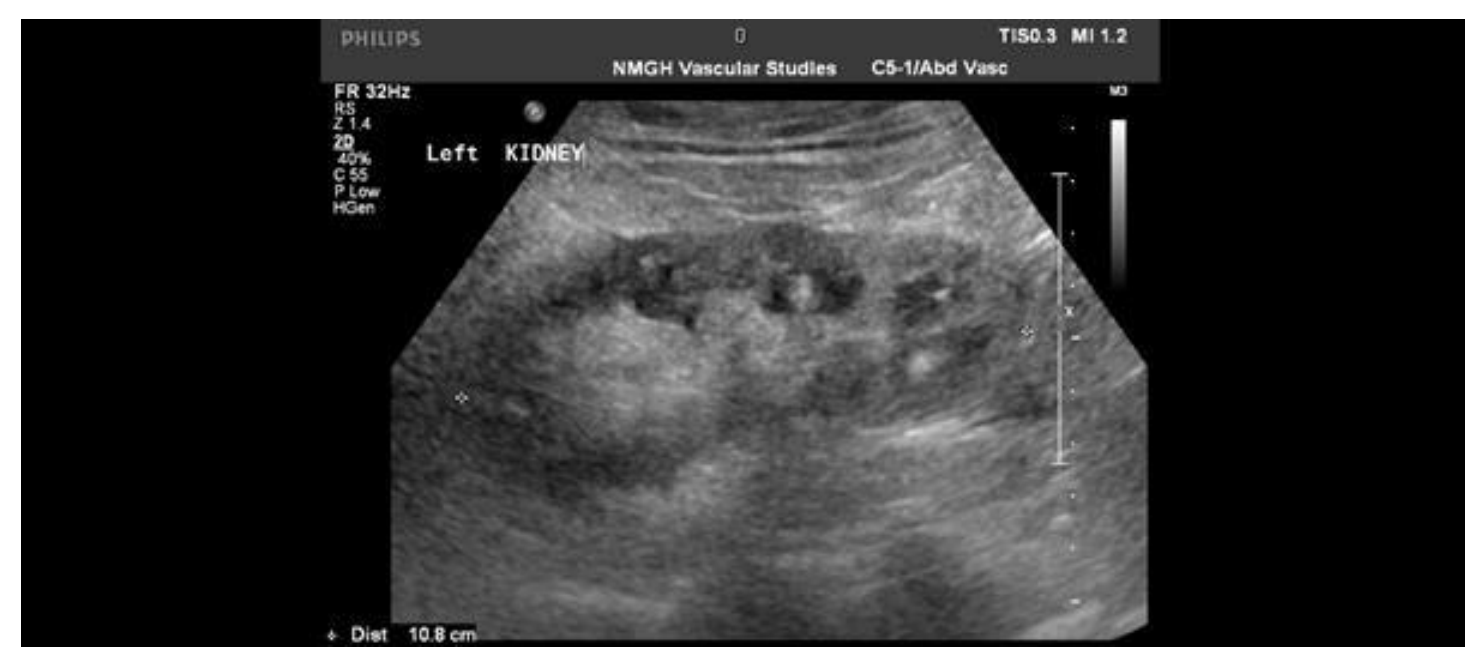

Figure: 6 KUB Vascular Ultrasound reports left artery thrombosis

\section{Discussion}

Infarction of renal artery is an infrequent condition which is very difficult to diagnose because of misleading clinical presentation. Similar clinical presentation is typically found in intestinal disease, aneurysm, valvular heart disease, fibromuscular disease, myocardial infarction, lymphoma, metastasis, muscle inflammation, genital disease, spinal disease and renal artery dissection (4).The source of renal artery thrombosis is found either blood source or cholesterol based emboli which cause partial occlusion with chronic presentation (5). In our patient echocardiogram excluded infective endocarditis and laboratory test for hypercoagulable cause of renal artery 
thrombosis. The common cause of renal artery thrombosis associated with arterial fibrillation, antiphospholipid syndrome, infective endocarditis, sickle cell disease, protein $S$ deficiency, protein $C$ resistance and trauma (6). Actual estimation of renal artery thrombosis is difficult because of rarity and uncommon clinical presentation. Deterioration of renal function more than $50 \%$ may be required to raise creatinine which could underestimate parenchymal damage (7). In general, patient presents with abdominal pain or flank pain associated with nausea and vomiting but occasional haematuria or fever also documented in different case studies. However, renal artery infarction may be completely asymptomatic which is incidentally identify during abdominal computed tomography (CT) originally requests for alternative diagnosis (8). Laboratory profile of elevated LDH suggests renal infarction in association with proteinuria, leucocytosis, haematuria with normal or minimally aminotransferase raise (9). The most sensitive markers for renal infarction is raised serum lactate dehydrogenase (LDH) despite few disease condition myocardial infarction, liver disease, cancer, muscle trauma and infection should be in consideration (10) Despite vague clinical presentation medical and surgical differential diagnosis were analysed carefully in this case study. The use of intravenous contrast during imaging study carefully assessed in view of concomitant renal impairment through risk and benefits rationale. In this patient our initial differential diagnosis were diverticulitis, gastroenteritis and even lower respiratory tract infection or ischaemic bowel based on clinical presentation. Initially our patient demonstrated no progress despite conventional management with antibiotics and intravenous fluids. Two days post admission confirmation of diagnosis was in dilemma because of no clinical and biochemically. Contrast enhanced CT was considered for evaluation of underlying pathology and exclusion of differential diagnosis despite some degree of renal impairment. We had noticed incidental findings of renal artery infarction by contrast enhanced CT scan which later supported by deteriorating renal function and raised lactate dehydrogenase. Unilateral renal artery thrombosis results acute kidney injury due to renal tubular necrosis as a result of renal hypoperfusion (11). Many case reports demonstrate contrast nephropathy related to acute kidney injury following angiography and contralateral kidney reflex vasospasm (12). In this case study, a contrast CT abdomen and pelvis confirmed renal artery thrombosis. Besides, non-invasive modality of radioisotope scan demonstrates decrease left renal perfusion. The diagnostic value for renal arterial duplex doppler demonstrates low sensitivity. The gold standard modality of investigation to diagnose renal artery thrombosis is renal arteriography (13). The treatment modalities for renal arterial thrombosis is anticoagulation with heparin followed by oral anticoagulants for long term. Patient should remain anticoagulation indefinitely where pro embolic condition remains within underlying medical history (14). The ideal way to treat New onset of hypertension or existing hypertension is ACEI or ARB which is mediated by elevation of renin (15). Thrombolysis and intra-arterial thrombectomy with or without angioplasty is an option for some selected patients which helps reperfusion and improvement of renal function. However, open surgery indicated for trauma associated renal infarction and thrombosis (16). 


\section{Conclusion}

Thromboembolic renal artery obstruction is not commonly seen in clinical practice which potentially may cause irreversible renal parenchymal damage. In non specific clinical setting of abdominal pain or flank pain renal artery thrombosis should be considered if triad of haematuria or proteinuria, risk of thromboembolism and raised serum LDH present. Recommendation for contrast enhanced CT to aide of diagnosis aimed at elevated LDH in this scenarios of atypical presentation. Besides, renal functional status follow up with evaluation of renal artery thrombosis and infarction should be achieved through renal radioisotope scan. The treatment modality based on early anticoagulation, thrombolysis and endovascular surgery in selective cases.

\section{Learning points}

-Clinical diagnosis of renal artery thrombosis with infarction not rare but demonstrations non-specific presentation.

-Renal infarction can be identified with triad of raised LDH, Haematuria or proteinuria, abdominal pain.

-Rapid diagnosis and treatment of renal artery thrombosis with infarction prevent irreversible renal parenchymal damage.

-Early diagnosis and appropriate intra-arterial fibrinolytic therapy or endovascular intervention restore renal function.

-Aim is to improve awareness in a situation clinician will contemplate early diagnosis and treatment.

\section{References}

1. Korzets Z, Plotkin E, Bernheim J et al, the clinical spectrum of acute renal infarction. IMAJ, 2002;4:781-4

2. Antopolsky M, Simanovsky N, Stalnikowicz R, Salameh S, Hiller N, Renal infarction in the ED: 10 year experience

3. Valerie M, Lopez, Jonathan Glauser, A case of renal artery thrombosis with renal infarction.

4. Demuynck F, Blanpain s, Brochart C, Morvan J, Boukadoum N, Fuks D, kocheida M, Saliou G, Yzet T, Remond A: Renal infarction: A rare manifestation of patent foranen ovale. J radiol, 2008, 89: 1101-1104

5. Frost L, Engholm G, Johnson S, Moller H, Henneberg EW, Husted S. Incident thromboembolism in the aorta and renal, mesenteric, pelvic, and extremity arteries after discharge from the hospital with a diagnosis of atrial fibrillation. Arch Intern Med. 2001; 161: 272-6

6. Lopez VM, Glauser J. A case of renal artery thrombosis with renal infarction. J Emerg Trauma Shock 2010; 3: 302

7. Syed MI, Shaikh A, Ullah A et al. Acute renal artery thrombosis treated with t-PA power-pulse spray rheolytic thrombectomy. Cardiovasc Revasc Med 2010; 11: 264.e1-264.e17 
8. Bande D, Abbara S, Kalva SP: Acute renal infarction secondary to calcific embolus from mitral annular calcification. Cardiovasc Intervent Radiol 2011, 34: 647-649

9. Domanovits $\mathrm{H}$, Paulis $M$, Nikfardjam $M$ et al. Acute renal infarction. Clinical characteristics of 17 patients. Medicine (Baltimore) 1999; 78 : 386-394

10. Oray NC, Bayram B, Yanturali S, et al. A potential pitfall in the diagnosis of renal colic: renal infarction. Am J Emerg Med 2009; 27; 750, E 7-9

11. Kuo-Liang Cheng, Shiou-Shan Tseng and Der-Cherng Tarng, Acute renal failure caused by unilateral renal artery thromembolism, Nephrol Dial Transplant, 2003, 18: 833-835.

12. Levin M, nakhoul F, Keidar Z, Green J. Acute oligouric renal failure associated with unilateral renal embolism: a successful treatment with iloprost. Am J Nephrol, 1998; 18: 444-447

13. Hazanov H, Somin M, Attali M et al. Acute renal embolism. Forty-four cases of renal infarction in patients with atrial fibrillation. Medicine (Baltimore) 2004; 83: 292-299

14. Hazanov H, Somin M, Attali M, Beilinson H, Thaler M, Moulallem M et al. Acute renal embolism. Fourty four cases of renal infarction in patients with atrial fibrillation. Medicine (Baltimore), 2004; 83: 292-9

15. Valerie ML, and Jonathan Glauer, A case of renal artery thrombosis with renal infarction. J Emerg Trauma and shock, 2010, 3(3).

16. LinHC, Shin PM, Chang TH, Ke HL, Wu WJ, Huang CH: Successful thrombolytic therapy for bilateral renal infarction: a case report. Kaohsiung J Med Sci 2006, 8:40 